\title{
In-situ Strain Analysis of Potential Habitat Composites Exposed to a Simulated Long-Term Lunar Radiation Exposure
}

\author{
Kristina Rojdev ${ }^{1,3}$, Mary Jane O’Rourke ${ }^{1}$, Charles Hill ${ }^{2}$, Steve Nutt ${ }^{3}$, William Atwell ${ }^{4}$ \\ ${ }^{1}$ NASA-Johnson Space Center, ${ }^{2}$ ESCG-Jacobs Engineering, ${ }^{3}$ University of Southern \\ California, ${ }^{4}$ The Boeing Company
}

One of NASA's missions is to continue to explore space beyond Low Earth Orbit (LEO) by focusing on technologies that will advance the state of the art and provide for longer duration missions. One area of interest is long-term surface habitation, which requires large structures to be in harsh environments for extended periods of time. Therefore, NASA is studying the effects of long-term space radiation on potential multifunctional composite materials for habitats and the focus of this paper is on strain and temperature data collected during high energy proton radiation exposure of two composite materials of interest for habitat structures.

The materials selected were a carbon fiber and epoxy material, which served as a baseline composite, and a boron and carbon fiber epoxy material, which was of interest as enhanced radiation shielding from secondary neutron exposure. The test setup simulated a material used as the primary structure of a cylindrical habitat pressure vessel. A test stand was designed and constructed to provide bi-axial tension on the composite samples, mimicking stresses on the material from an internal pressure environment. The samples contained a bi-axial strain gauge to gather strain data in both axes and a thermocouple to gather temperature readings of the sample. There was also a thermocouple placed in the chamber to understand sample temperature correlation to the air temperature. The samples were irradiated at Indiana University with a $200 \mathrm{MeV}$ proton exposure of 500 krads to simulate a 30 year mission. In addition, samples were irradiated at a slow dose rate of thirteen hours, or at a fast dose rate of one hour to investigate whether accelerated radiation exposure had an effect on the material.

The results showed that the slow dose rate exposure increased the sample strain over time in both axes, whereas the fast dose rate exposure decreased the sample strain over time in both axes. While the strain changes were small, on the order of microstrain, the trend in the data was consistent between the different runs. In addition, the thermocouples showed a slight increase in temperature, corresponding to the chamber air temperature, during the slow dose rate runs. During the fast dose rate exposures there was an overall decrease in temperature, also in accordance with the chamber temperature. The changes in temperature are most likely due to the warming and cooling of the building throughout the day.

Given that the increase or decrease in strain seemed to correspond with the temperature data collected, additional calculations were performed to see if the strain changes in the material were due to the temperature changes. It was found that the temperature data could not be correlated to the strain changes, and it was concluded that the strain changes were due to the radiation exposure. Thus, the results suggest that a slow dose rate exposure stretches the material, possibly due to chain scission occurring in the matrix. However, the fast dose rate exposure shows decreasing strain, suggesting shrinkage of the matrix, possibly due to cross-linking, or post-curing occurring.

In summary, two composite materials were exposed to a simulated 30 year radiation exposure while simultaneously undergoing bi-axial tensile stress to mimic internal pressure stresses of a habitat pressure vessel. The results showed that radiation exposure affects the strain of a material during exposure, and the type of effect is dependent on the rate of exposure. Further characterization will be performed on these samples to validate the mechanism by which the materials changed. 


\title{
In-Situ Strain Analysis of Potential Habitat Composites Exposed to a Simulated Long-Term Lunar Radiation Exposure
}

\author{
Kristina Rojdev, \\ NASA-Johnson Space Center, 2101 NASA Parkway, Houston, TX 77058 \\ kristina.rojdev-1@nasa.gov \\ University of Southern California, 854B Downey Way, Los Angeles, CA 90089 \\ rojdev@usc.edu \\ Mary Jane O’Rourke, \\ NASA-Johnson Space Center, 2101 NASA Parkway, Houston, TX 77058 \\ Maryjane.e.orourke@nasa.gov \\ Charles Hill, \\ NASA-Johnson Space Center, 2101 NASA Parkway, Houston, TX 77058 \\ Charles.Hill2@escg.jacobs.com \\ Steve Nutt, \\ University of Southern California, 3651 Watt Way, VHE 406, Los Angeles, CA 90089 \\ nutt@usc.edu \\ And \\ William Atwell \\ The Boeing Company, 13100 Space Center Blvd., HB 2-30, Houston, TX 77059 \\ william.atwell@boeing.com
}

\begin{abstract}
NASA is studying the effects of long-term space radiation on potential multifunctional composite materials for habitats to better determine their characteristics in the harsh space environment. Two composite materials were selected for the study and were placed in a test stand that simulated the stresses of a pressure vessel wall on the material. The samples in the test stand were exposed to radiation at either a fast dose rate or a slow dose rate, and their strain and temperature was recorded during the exposure. It was found that during a fast dose rate exposure the materials saw a decreased strain with time, or a shrinking of the materials. Given previous radiation studies of polymers, this is believed to be a result of crosslinking occurring in the matrix material. However, with a slow dose rate, the materials saw an increase in strain with time, or a stretching of the materials. This result is consistent with scission or degradation of the matrix occurring, possibly due to oxidative degradation.
\end{abstract}

\section{Keywords}

Composites; proton; radiation; strain; crosslinking; scission 


\section{Introduction}

One of NASA's missions is to continue to explore space beyond Low Earth Orbit (LEO) by focusing on technologies that will advance the state of the art and provide for longer duration missions. One area of interest is long-term surface habitation, which requires large structures to be in harsh environments for extended periods of time. These large structures need to be lightweight and multifunctional in nature. Thus, polymeric composites have gained interest as a potential structural material for surface habitation. However, there are still several unknowns in using composite materials in a space environment, and specifically a long-term radiation environment. To better understand this problem, NASA is studying the effects of long-duration radiation exposure on potential multifunctional composite materials for habitats. The following paper discusses the strain and temperature response of two composite materials during radiation and bi-axial exposure, simulating a habitat on the lunar surface during a long-duration exposure to radiation.

\subsection{Assumptions}

In performing this work, certain assumptions were made to constrain the study. This work assumes a habitat is on the lunar surface and in service for thirty years. Even though the lunar surface is chosen for this study, any planetary surface with limited atmosphere and magnetic field would be applicable with regards to the radiation environment. In addition, it is assumed the habitat is pressurized with air at an elevated oxygen concentration and is unshielded from the radiation environment on the exterior. Finally, it is assumed that the habitat is exposed to one very large solar particle event (SPE) during each solar cycle, as well as a constant galactic cosmic ray (GCR) exposure.

\subsection{Space Radiation Environment}

There are two primary forms of space radiation that are of concern for materials: Solar Particle Events (SPE) and Galactic Cosmic Rays (GCR). The SPEs emanate from the sun, which follows an eleven year solar cycle. During a period known as solar maximum, the sun is very active and tends to have more SPEs than during solar minimum. These SPEs range in intensity and in frequency, as shown in Figure 1.

The GCRs are very high energy radiation penetrating the heliosphere from outside the solar system. Every element in the periodic table is included in the GCR particles, and the protons are the most abundant. However, the concern with GCRs typically results from the prevalence of heavy ions, such as iron. Unlike SPEs, the GCRs are an ever present background radiation and are isotropic within the solar system, yet they are still modulated by the solar cycle such that the GCR intensity is greatest during solar minimum.

\subsection{Preliminary Environmental Modeling}

To better understand the environment in which these materials would be exposed, and to develop a baseline for the experimental radiation, some initial modeling was performed. In Figure 2, the absorbed dose on a material from GCRs versus SPEs was compared, showing that the majority of 
absorbed dose is a result of SPE exposure. Thus, the following study focused on proton radiation with characteristics similar to that of an SPE. In Figure 3, the absorbed doses for three different mission lengths are compared. The total absorbed dose displayed in this figure is from the addition of the GCR exposure and the SPE exposure, which is then multiplied by a factor of safety of ten. The factor of safety is to add in any additional SPE exposure that may have occurred during the solar cycle, and is not necessarily a very large SPE. For the worst case scenario, a 30 year mission would encounter approximately 500,000 cGy of exposure, based on these calculations.

\subsection{Habitation Calculations}

The internal pressure environment of the habitat set by NASA in conjunction with the lunar environment has specific design implications on the habitat (Jablonski, 2008). Due to the lack of atmosphere on the lunar surface and the internal pressure of the habitat, the predominant stress on the habitat while on the lunar surface is the internal pressure. This stress is manifested as biaxial tensile stress on the material of the pressure vessel. Therefore, to consider a realistic worst-case scenario, a skin-stiffened laminate was selected to represent the habitat shell. Given the minimum gage thickness of a skin-stiffened structure (Dorsey, 2008) and using the calculations for normal and hoop stress on a pressure vessel, the worst case stress imparted to a cylindrical habitat due to internal pressure was calculated to be approximately $41 \mathrm{MPa}$.

2. Experimental

\subsection{Materials}

There are two materials that were used for this study. The first material is a pre-impregnated composite made of Hexcel IM7 carbon fibers and Cycom 977-3 toughened-epoxy resin. The second material is a pre-impregnated composite from Specialty Materials, Inc. that is made of 4mil boron fibers and MR40 carbon fibers impregnated with Newport 301 toughened-epoxy resin. All samples were manufactured in-house with a layup design of [+60/-60/0 $]_{s}$. The IM7 samples were cure in an autoclave, whereas the Hybor samples were cured in a press. Both methods of cure followed the recommended cure cycles provided by the manufacturer.

\subsection{Test Setup}

Prior to radiation exposure, the samples were placed in a test stand that provided bi-axial tension on the samples. In addition, they had a bi-axial strain gauge placed on the center of the sample and a thermocouple placed close to the strain gauge. The strain gauge leads and some of the thermocouples were connected to a National Instruments Data Acquisition System. Other thermocouples were connected to hand-held readers placed in the beam room. Web cameras were used to acquire the readings off of the hand-held readers. The data that was collected during the radiation exposures were the change in strain in both directions of the sample and temperature of the sample surface. 


\subsection{Radiation Exposures}

All samples were exposed to a total dose of 500 krads using protons of $200 \mathrm{MeV}$ energy. However, samples were either exposed to a fast dose rate of $0.1478 \mathrm{krad} / \mathrm{s}$ or a slow dose rate of $0.0139 \mathrm{krad} / \mathrm{s}$. The exposures were performed at Indiana University Cyclotron Facility.

There were a total of six runs completed for this experiment. All the samples for each run were stacked in front of the radiation beam. The following table shows the conditions for each run.

\begin{tabular}{l|ccc} 
Run \# & Dose Rate & \# of Samples & Material \\
Run 1 & Slow & 5 & Hybor \\
Run 2 & Slow & 5 & IM7 \\
Run 3 & Fast & 1 & IM7 \\
Run 4 & Fast & 1 & IM7 \\
Run 5 & Slow & 4 & 2 - Hybor, 2 - IM7 \\
Run 6 & Fast & 4 & 2 - Hybor, 2 - IM7 \\
\hline
\end{tabular}

2.4. Calculations of material strain due to thermal changes

When the temperature data collected was compared with the strain data collected for each sample, it was noticed that the temperature trends for each run were similar to the strain trends of the respective runs. Thus, calculations were carried out to compare thermal strains with the strain data collected during the radiation runs.

First, the thermal strain change of the material sample was calculated, using the following equation. Here, $\alpha_{\mathrm{lam}}$ is the coefficient of thermal expansion for the material sample and $\Delta \mathrm{T}$ is the change in temperature.

$$
\Delta_{\text {Strain_lam }}=\alpha_{\text {lam }} \Delta T
$$

Then the thermal strain change of the test stand was calculated. The outer ring of the test stand is made of aluminum, and the equation for the test stand is the following equation. Here, $\alpha_{\mathrm{Al}}$ is the coefficient of thermal expansion for the aluminum frame and $\Delta \mathrm{T}$ is the change in temperature.

$$
\Delta_{\text {Strain_Al }_{A}}=\alpha_{A l} \Delta T
$$

After these two calculations were completed, the change in strain between the two equations was compared and the greater value was assumed to be the driving force for the strain measurements observed. These calculations were then compared with the change in strain measured on the samples during radiation exposure.

3. Results

\subsection{Fast Dose Rate Samples}

The samples that were exposed to a fast dose rate were part of Run 3, Run 4, and Run 6 . All of the samples in each of these runs had an overall decrease in strain with respect to time, in both 
axes. Using Run 6 as an example, the decreasing strain was apparent in both materials, as shown in Figure 5. In Figure 6, the corresponding temperature data collected for these materials is shown. Both of these graphs correspond to data collected during Run 6 in which two Hybor samples (Hybor-9 and Hybor-10) were stacked with two IM7 samples (IM7-1 and IM7-2). In the temperature data, IM7-1 did not get recorded due to a hardware malfunction.

In both the strain and temperature data, there is a general decreasing trend that is very similar. To better understand whether the strain in the sample was due to radiation or thermal induced strains, calculations of thermal strain were completed for both the aluminum frame and the sample. For the Hybor material, an example of these calculations is shown in Figure 7 and for the IM7 material, an example of these calculations is shown in Figure 8. For both materials, it is shown that the Aluminum frame would expand more than the sample, and would thus be the leading cause for strain in the sample. However, the calculated strain due to thermal expansion of the Aluminum frame does not match the strain measured in the sample during the radiation exposure.

\subsection{Slow Dose Rate Samples}

In Run 1, Run 2, and Run 5, samples were exposed to a slow dose rate. These samples showed a trend towards increasing strain with respect to time. Using Run 1 as an example, Figure 9 shows several Hybor samples with an increased strain with respect to time in both axes, followed by a gradual leveling out of the data towards the latter part of the run. In this figure there are also two dashed lines that do not follow the rest of the data and correspond to data recorded from Hybor6B and Hybor-11B. These two axes did not record any data for Run 1, most likely due to software and hardware failures from radiation exposure.

Following a similar trend, the temperature data for these samples is shown in Figure 10. While the graphs of Figure 9 and Figure 10 are initially dissimilar, they both tend to trend upwards throughout the run. Thus, calculations were performed to see if the thermal expansion of the Aluminum frame or the thermal expansion of the sample itself was causing the measured strain. The plot of these calculations overlaid with the measure data is shown in Figure 11 for the sample Hybor-4. In this plot, the expansion due to the aluminum frame is much greater than the thermal expansion of the sample. So, if the sample was expanding due to thermal forces, the aluminum frame would dominate the expansion. However, the measured strain does not match that of the aluminum frame.

A similar trend is seen in the IM7 material (Figure 12) prior to 30,000 seconds when considering IM7-9 that was exposed to a slow dose rate during Run 2. In this figure, there is a break in the data from approximately 30,000 seconds to 38,000 seconds due to a failure in the software, most likely from the radiation exposure. When the software was restarted, the strain was recalibrated which is the reason for the jump in the strain data back to zero. Had the strain not been recalibrated, the strain data would have most likely shown a leveling out of the strain at around $5.25 \mathrm{E}-5$ in/in.

\section{Discussion}


In this study it was found that at a fast dose rate exposure, samples exhibited a decrease in strain with respect to time, whereas with a slow dose rate exposure, samples exhibited an increase in strain with respect to time. It was also determined through calculation that these results were not due to thermal changes in either the test stand providing tension on the samples or the samples themselves. In addition, both materials exhibited these properties during the radiation exposures, removing the possibility that these effects are material specific for the materials studied. Thus it is believed that these strain changes are a result of the radiation exposure on the materials.

In several previous studies and articles (Al-Sheikhly, 1994 more references), it has been shown that there are two main mechanisms that occur to polymeric materials exposed to radiation: chain scission and crosslinking. Generally, these two mechanisms occur simultaneously with one predominating over the other. Crosslinking typically results in increased strength, increased average molecular weight, and embrittlement of the matrix whereas chain scission results in decreased strength, decreased molecular weight, and degradation of the matrix (i.e. ductility).

Comparing the outcome of these two mechanisms with the measured strain data during the radiation exposure, it seems as if with a fast dose rate exposure, the materials are shrinking possibly due to enhanced crosslinking of the matrix. However, with a slow dose rate exposure, the samples are stretching possibly due to degradation of the matrix through scission. A similar observation was also found in a study by K. Gillen and R. Clough (1981b) where dose rate effects existed for four different materials. It was concluded that scission effects became more important as the dose rate decreased. In addition, it was found that oxidative degradation was a possible explanation for the scission dominance at decreased dose rates. Other studies (Briskman, et al. 2004, Gillen and Clough, 1981a, Gillen and Clough, 1989, Sasuga, et al., 1985, and Seguchi, et al., 1981) have also discussed similar findings of scission related effects due to oxidation at low dose rates. Given that this study is performed in air at two extreme dose rates, it is possible that the strain effects are also due to oxidative degradation occurring more heavily in the slow dose rate samples, giving rise to the increased strain behavior. Further characterization of these materials will need to be completed in the future to validate these mechanisms.

5. Summary and Conclusions

Two composite materials were evaluated in a long-term radiation environment at two different dose rates. These materials were also subjected to a simulated pressure stress while being exposed to radiation, and the strain of the materials was recorded during the exposure. It was found that with a fast dose rate, the materials decreased in strain, a shrinking of the material, and with a slow dose rate, the materials increased in strain, a stretching of the material. It was also concluded that the strain changes observed in the samples were due to the radiation exposure, and not thermally induced strain changes. Finally, comparing the measured results with previous studies of radiation exposed polymeric materials shows a similar trend in that scission is more dominant with a decreased radiation dose rate, and is potentially due to oxidative degradation. 


\section{Acknowledgements}

The authors would like to acknowledge the guidance and resources provided by NASA's Materials and Processes branch in the development and analysis of this study. In addition, we would like to thank the Avionics System Division for their support with the irradiation of samples and data collection hardware borrowed for this study.

\section{References}

Al-Sheikhly, M., Christou, A., 1994. Tutorial: How Radiation Affects Polymeric Materials. IEEE Transactions on Reliability. 43 (4), 551-556.

Briskman, B.A., Klinshpont, E.R., Stepanov, V.F., Tlebaev, K.B., 2004. Determination of Dose Rate Effects in Polymers Irradiated in Vacuum. Journal of Spacecraft and Rockets. 41(3), 360-365.

Dorsey, J.T., Wu, K.C., Smith, R., 2008. Structural Definition and Mass Estimation of Lunar Surface Habitats for the Lunar Architecture Team Phase 2 (LAT-2) Study. Proceedings of Earth and Space 2008.

Gillen, K.T., Clough, R.L., 1981a. Radiation-Thermal Degradation of PE and PVC: Mechanism of Synergism and Dose Rate Effects. Radiat. Phys. Chem. 18 (3-4), 661-669.

Gillen, K.T., Clough, R.L., 1981b. Occurrence and Implications of Radiation Dose-Rate Effects for Material Aging Studies. Radiat. Phys. Chem. 18 (3-4), 679-687.

Gillen, K.T., Clough, R.L., 1989. Time-Temperature-Dose Rate Superposition: A Methodology for Extrapolating Accelerated Radiation Aging Data to Low Dose Rate Conditions. Polymer Degradation and Stability. 24, 137-168.

Jablonski, A.M., Ogden, K.A., 2008. Technical Requirements for Lunar Structures. Journal of Aerospace Engineering. 2, 72.

Rose, R., 2008. Natural and Induced Environments. Man-Systems Integrated Standards. Online. Internet. 15 March 2009. Available: http://msis.jsc.nasa.gov/sections/section05.htm

Sasuga, T., Hayakawa, N., Yoshida, K., Hagiwara, M., 1985. Degradation in Tensile Properties of Aromatic Polymers by Electron Beam Irradiation. Polymer. 26, 1039-1045.

Seguchi, T., Arakawa, K., Hayakawa, N., Machi, S., 1981. Radiation Induced Oxidative Degradation of Polymers - IV. Dose Rate Effects on Chemical and Mechanical Properties. Radiat. Phys. Chem. 18 (3-4), 671-678. 